\title{
Challenge Agent Treatment Efficacy Study
}

National Cancer Institute

\section{Source}

National Cancer Institute. Challenge Agent Treatment Efficacy Study. NCI Thesaurus.

Code C158465.

A study that assesses the efficacy of treatment given after a protocol-defined manifestation of the challenge agent(s)-induced disease or condition. 